\title{
Singularity aware de-homogenization for high-resolution topology optimized structures
}

Stutz, F. C.; Groen, J. P.; Sigmund, O.; Bærentzen, J. A.

\section{Published in:}

Structural and Multidisciplinary Optimization

Link to article, DOI:

10.1007/s00158-020-02681-6

Publication date:

2020

Document Version

Peer reviewed version

Link back to DTU Orbit

Citation (APA):

Stutz, F. C., Groen, J. P., Sigmund, O., \& Bærentzen, J. A. (2020). Singularity aware de-homogenization for high-resolution topology optimized structures. Structural and Multidisciplinary Optimization, 62, 2279-2295. https://doi.org/10.1007/s00158-020-02681-6

\section{General rights}

Copyright and moral rights for the publications made accessible in the public portal are retained by the authors and/or other copyright owners and it is a condition of accessing publications that users recognise and abide by the legal requirements associated with these rights.

- Users may download and print one copy of any publication from the public portal for the purpose of private study or research.

- You may not further distribute the material or use it for any profit-making activity or commercial gain

- You may freely distribute the URL identifying the publication in the public portal 


\title{
Singularity Aware De-Homogenization for High-Resolution Topology Optimized Structures
}

\author{
F. C. Stutz ${ }^{1}$ • J. P. Groen ${ }^{2}$ • O. Sigmund ${ }^{2}$ • J. A. Bærentzen ${ }^{1}$
}

Received: date / Accepted: date

\begin{abstract}
Homogenization-based topology optimization has been shown to be effective but does not directly create mechanical structures. Instead, the method gives a multi-scale description of the optimized design e.g. lamination thicknesses and directions. To obtain a realizable single-scale design one can perform a subsequent de-homogenization step. This is done by converting the lamination directions to integrable vector fields from which it is possible to compute a parameterization of the domain. Unfortunately, however, singularities often make it impossible to find integrable vector fields that align with lamination directions. We present a short introduction to homogenization-based topology optimization followed by an overview of different types of singularities and how they impinge on the problem. Based on this, we propose a singularity aware de-homogenization pipeline, where we use a method for vector field combing which produces consistent labeling of the lamination directions but also introduces necessary seams in the domain. We demonstrate how methods from computer graphics can subsequently be used to compute the final parameterization from which the mechanical structure can easily be extracted. We demonstrate the method on several test cases.
\end{abstract}

Keywords De-homogenization - Singularities · Topology Optimization · High Resolution Structures

F. C. Stutz ${ }^{1}$. J. P. Groen ${ }^{2}$. O. Sigmund ${ }^{2}$. J. A. Bærentzen ${ }^{1}$ ${ }^{1}$ Department of Applied Mathematics and Computer Science ${ }^{2}$ Department of Mechanical Engineering, Technical University of Denmark, 2800 Kgs. Lyngby, Denmark

E-mail: fstu@dtu.dk

\section{Introduction}

Topology optimization, a numerical tool for determining optimal mechanical layouts, has a broad spectrum of possible industrial applications. Over the last decades, the development and accessibility of computational power have made it an interesting design tool for the industry. However, further developments are still needed before large-scale, real-time topology optimization is possible on desktop computers.

Homogenization-based topology optimization, as proposed by Bendsøe and Kikuchi (1988), allows for composite material properties, which contain much more information compared to the isotropic material used in the SIMP (Solid Isotropic Material with Penalisation) approach (Bendsøe, 1989). Hence, performing homogenization-based topology optimization allows us to solve the problem on a coarse grid while still enabling near-optimal, high-resolution results as shown in Groen and Sigmund (2018). In homogenization-based topology optimization, we can describe the composite material as infinitesimally small periodic unit cells on a microscopic level. On a macroscopic level, we can assume the material properties to be homogeneous. Hence, we can analyze the performance of a design where these composite properties are spatially varying in a multi-scale sense.

A subset of problems of interest in topology optimization is the compliance minimization of a plane structure subject to a single load case, which is considered in this paper. For these types of problems, it is known that hierarchical rank-2 laminates are the optimal material parameterization (Allaire and Kohn, 1993). Nevertheless, we use the square unit-cell with a rectangular hole as proposed by Bendsøe and Kikuchi (1988) since these microstructures consist of a single 
length-scale and have only a small difference in performance (Bendsøe and Sigmund, 1999, Träff et al., 2019).

The output from the single load homogenizationbased optimization method can be interpreted as a cross-field: at each point of the optimized domain (in $2 \mathrm{D}$ ), we have a pair of directions (as well as lamination thicknesses). Ultimately, our goal is to create a mechanical design whose structural members are well aligned with these frame directions. A particular approach to this problem involves creating a parametrization of the domain such that the gradients of the coordinate functions in the parametric domain are aligned with the cross-field obtained from the optimization procedure. If we are able to do that, it is possible to create a regular structure in the parametric domain and map it via the inverse parametrization back into the domain of the optimization. This procedure is known as the de-homogenization method (Pantz and Trabelsi, 2008; Groen and Sigmund, 2018, Donders, 2018, Groen et al. 2020).

There are different ways to optimize the unit-cell orientation of spatially varying designs. For instance, it is well-known that for 2- and 3-dimensional single load case examples a unit-cell is optimally aligned using the principal stress directions (Pedersen, 1989, Norris, 2005). However, the principal stress directions are not consistently labeled. Only in 2D can the stress directions be sorted to be smooth up to 180 degrees, see e.g. Groen and Sigmund (2018). Similarly, when the unit-cell orientations are optimized using gradientbased optimization, jumps of 90 degrees can occur. In other words, the first lamination direction at one point might correspond to the second lamination direction at a nearby point.

Recently, Groen and Sigmund (2018) simplified and further developed the projection approach by Pantz and Trabelsi (2008). However, this approach relied on two smooth vector fields (see Definition 2, 1-vector fields). They showed that these can be obtained by using orientations based on the principal stress direction. Similar to these directions the orientation of the microstructure is rotationally symmetric by 180 degrees, i.e. the 1-vector fields often contain jumps of angle $\pi$. Groen and Sigmund (2018) showed that this problem can be solved by using connected component labeling in case there is no singularity present.

\subsection{Contributions}

In this paper, we would like to investigate how integration approaches can be extended for the usage in fields containing singularities. This has been researched in Pantz and Trabelsi (2010) and Donders (2018). The main contribution of this paper is a pipeline for the de-homogenization of structures containing singularities as they occur for example due to loads inside the domain, but also due to numerically unstable results in low-density areas and regularization. Here, we use the word de-homogenization for the process of extracting a mechanically well-performing structure from the solution of the homogenization method. The pipeline allows us to investigate the location and type of singularities that appear in fields arising from the homogenizationbased topology optimization method, and their relation to the density distribution. We compare different starting guesses for the homogenization-based topology optimization and their influence on the resulting structure. Furthermore, we will investigate how to extract two as smooth as possible vector fields in the presence of singularities. This extraction of two vectors per element is called combing in computer graphics and is normally done by using a breadth-first search: Starting from a root element all elements in the domain are visited in order of growing distance to the root element (see further in Section 5.2, Skiena (2008)). We will use a density-based approach, which allows for simpler seams (see Definition 5, seams) than in the case of using a breadth-first search. We then relate the parametrization step to a computer graphics method for quadrilateral meshing (Bommes et al. (2009)), by introducing seams in the parameterization domain in order to allow for mismatches caused by the singularities, while still getting smooth de-homogenized designs.

In general, we specify parameters, running times and compliance values in order to allow the reader to observe the influence of these parameters to the dehomogenized structure and motivate further research areas. It is not our primary goal in this work to tune the parameters of the pipeline for mechanical performance.

\section{Related work}

Pantz and Trabelsi (2008) proposed a method to project the unit square microstructure macroscopically. Their de-homogenization approach minimizes an alignment energy to find a parametrization aligned with the orientation of the microstructure. The approach assumes two separate vector fields, whose orientation is not of importance. Groen and Sigmund (2018) simplified the approach by Pantz and Trabelsi, still using two separate vector fields, however, with the challenge of having consistently aligned vector fields as the approach is not invariant to angle jumps of angle $\pi$. This was solved by using a connected component analysis on the vector fields. Both approaches do not explicitly deal with 
jumps of angle $\pi / 2$. Pantz and Trabelsi (2010) investigated the challenge of singularities for the first time. The singularities, located in void regions, are nucleated, i.e. the areas containing singularities are excluded from the parametrization step and onwards. Donders (2018) proposed a method of spotting singularities of index $\pm 1 / 2$ using a technique explained in more detail in Hotz et al. (2010). This only allows for jumps of angle $\pi$. In order to deal with the singularities, correction functions are computed to prevent mismatches at seams.

The work of Kälberer et al. (2007) proposed the "Quad-Cover" algorithm for field-guided quad meshing using multiple coverings. Multiple coverings in the sense that the parametrization domain is duplicated multiple times. This means, that the rotationally symmetric field simplifies to vector fields on every single covering and thus becomes integrable. In practice only a single parametrization domain is used and operators to account for the different coverings are introduced. Bommes et al. (2009) proposed a refinement of QuadCover, solving the resulting system in an iterative approach yielding better results.

\section{Homogenization-based topology optimization}

The methodology to perform homogenization-based topology optimization used here is very similar to the method in Groen and Sigmund (2018), and therefore this section will be kept brief, with an emphasis on the differences.

\subsection{Microstructure parameterization}
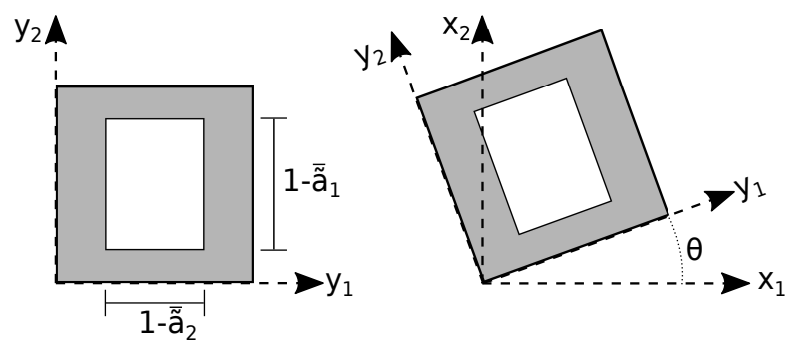

Fig. 1: Layout of the unit-cell with a rectangular hole, in local $\left(\mathrm{y}_{1}, \mathrm{y}_{2}\right)$, and global $\left(\mathrm{x}_{1}, \mathrm{x}_{2}\right)$ coordinate system.

As a microstructure parameterization, we use the square unit-cell with a rectangular hole proposed by Bendsøe and Kikuchi (1988) and shown in Figure 1. The constitutive properties of this microstructure, consisting of void and isotropic material using $E=1$ and $\nu=0.3$, can be obtained using numerical homogenization on a conformal mesh. A database of the constitutive properties in the local frame can be pre-computed for various physical heights $\overline{\tilde{a}}_{1}$ and physical widths $\overline{\tilde{a}}_{2}$ of the hole. Afterwards, we can interpolate the effective properties $\boldsymbol{E}^{H}$ and corresponding sensitivities for any combination of $\overline{\tilde{a}}_{1}$ and $\overline{\tilde{a}}_{2}$. The elasticity tensor in the global frame of reference $\boldsymbol{E}$ can then be obtained as,

$\boldsymbol{E}\left(\theta, \overline{\tilde{a}}_{1}, \overline{\tilde{a}}_{2}\right)=\boldsymbol{R}(\theta) \boldsymbol{E}^{H}\left(\overline{\tilde{a}}_{1}, \overline{\tilde{a}}_{2}\right) \boldsymbol{R}(\theta)^{\mathrm{T}}$,

with $\boldsymbol{R}$ being the rotation matrix. The volume fraction of the microstructure $\rho$ is given as,

$\rho=1-\overline{\tilde{a}}_{1} \overline{\tilde{a}}_{2}$

3.2 Optimization and regularization of the

microstructure orientation

Contrary to the approach presented in Groen and Sigmund, 2018) we update the angles based on their gradients instead of the principal stress directions. There are two reasons for this. First, updating the angles based on the gradients is slightly more stable than updating the orientation based on the principal stress directions (Pedersen, 1989), since first-order finite elements are used. Second, and more importantly, we can introduce a regularization energy, similar to the one in Groen et al. 2020) such that the 4-direction field is slowly varying over the domain.

To perform the regularization we loop over all $n_{e}$ edges, each connecting two elements. We introduce a penalization function $\mathcal{P}_{e} \in[0,1]$. This penalization function is based on the orientation angles of the microstructure in the two adjacent elements $f_{1}$ and $f_{2}$ connected by edge $e$. We denote these two angles with $\theta_{f_{1}}$ and $\theta_{f_{2}}$ and calculate

$\mathcal{P}_{e}=\frac{1}{2}-\frac{1}{2} \cos \left(4 \theta_{f_{1}}-4 \theta_{f_{2}}\right)$.

It can be seen that $\mathcal{P}_{e}$ has minimum values at angle differences of 0 or $k \pi / 2$ with $k$ an integer and returns a small penalty value for small angle differences. By looping over all edges $e$ we obtain a single regularization objective $\mathcal{F}_{\theta}$,

$\mathcal{F}_{\theta}=\sum_{f=1}^{n_{e}} \mathcal{P}_{e}$,

which is normalized in the optimization problem using the regularization objective $\mathcal{F}_{\theta}^{(1)}$ for the starting guess of the layer-normals. 


\subsection{Problem formulation}

We focus on minimizing the objective functional $\mathcal{F}$, which is a combination of the compliance $\mathcal{J}$ and regularization objective $\mathcal{F}_{\theta}$. The domain is discretized using bi-linear finite elements and the material properties are assumed to be element-wise constant. We want to avoid checkerboard-like instabilities that can occur due to the use of bi-linear finite elements (Díaz and Sigmund, 1995). Therefore, we use a standard density filter to obtain the filtered hole height $\tilde{\boldsymbol{a}}_{1}$ and width $\tilde{\boldsymbol{a}}_{2}$ from design vectors $\boldsymbol{a}_{1}$ and $\boldsymbol{a}_{2}$ (Bourdin, 2001; Bruns and Tortorelli, 2001).

Furthermore, we want the microstructure widths to be either void, completely solid or in the interval $[\eta, 1-$ $\eta$ ], with $\eta=0.05$ used in this study. To do so, we use the same interpolation scheme as in Groen and Sigmund, 2018, Groen et al. 2020), which modifies the variables $\tilde{\boldsymbol{a}}_{1}$ and $\tilde{\boldsymbol{a}}_{2}$ into the physical hole height $\overline{\tilde{\boldsymbol{a}}}_{1}$ and width $\overline{\tilde{\boldsymbol{a}}}_{2}$. The optimization problem is solved in nested form, which means that for each design iteration we solve the state equation after which the design vectors are updated. Hence, the discretized optimization problem can be written as,

$$
\begin{aligned}
\min _{\boldsymbol{a}_{1}, \boldsymbol{a}_{2}, \boldsymbol{\theta}}: & \mathcal{F}\left(\boldsymbol{a}_{1}, \boldsymbol{a}_{2}, \boldsymbol{\theta}, \mathbf{U}\right)= \\
& \frac{\mathcal{J}\left(\boldsymbol{a}_{1}, \boldsymbol{a}_{2}, \boldsymbol{\theta}, \mathbf{U}\right)}{\mathcal{J}^{(1)}}+\gamma_{\theta} \frac{\mathcal{F}_{\theta}(\boldsymbol{\theta})}{\mathcal{F}_{\theta}^{(1)}}, \\
\text { s.t. } & \mathbf{K}\left(\boldsymbol{a}_{1}, \boldsymbol{a}_{2}, \boldsymbol{\theta}\right) \mathbf{U}=\mathbf{F}, \\
& : \mathbf{v}^{T} \boldsymbol{\rho}\left(\boldsymbol{a}_{1}, \boldsymbol{a}_{2}\right)-V_{f}^{\max } A \leq 0, \\
& : \mathbf{0} \leq \boldsymbol{a}_{1}, \boldsymbol{a}_{2} \leq \mathbf{1}, \\
& : \mathbf{- 4} \boldsymbol{\pi} \leq \boldsymbol{\theta} \leq \mathbf{4 \pi},
\end{aligned}
$$

where $\mathbf{v}$ is the vector containing the element areas and $V_{f}^{\max }$ is the maximum allowed fraction of the material in $\Omega$, with $A$ the area of $\Omega$. $\mathbf{K}$ is the stiffness matrix and vector $\mathbf{F}$ describes the loads acting on the domain. $\mathcal{J}^{(1)}$ is the compliance of the first analysis step and $\mathcal{F}_{\theta}^{(1)}$ the regularization objective for the starting guess. For the design update we use the MATLAB implementation of the Method of Moving Asymptotes (MMA) introduced by Svanberg (1987). Finally, it should be mentioned that the compliance minimization problem is self-adjoint, meaning that we only require the solution of one linear system to obtain both compliance and corresponding sensitivities.

As a starting guess for the microstructure we use $\boldsymbol{a}_{1}=\boldsymbol{a}_{2}$, such that the volume constraint is exactly satisfied. The starting guess for the orientation is based on a pre-analysis using isotropic microstructures, the corresponding principal stress directions are used to determine $\boldsymbol{\theta}$ unless otherwise noted. Finally, it should be mentioned that the scaling parameter $\gamma_{\theta}$ has a large influence on the optimization procedure and is in general chosen to be $\gamma_{\theta}=1 / 20$.

\section{Singularities}

In order to study the vector fields generated by the homogenization method, it is important to have a general understanding of vector fields, direction fields and the eigenvectors and eigenvalues of the stress tensors. This allows us to precisely define singularities and their indices and use this knowledge to study stress-tensor and layer-normal fields. Vaxman et al. (2016) introduced a notation that is tailored for this purpose. We will in the following section describe relevant definitions from Vaxman et al. (2016) related to the homogenization method and introduce their notation. We start with the definition of a directional field and $N$-directional fields.

Definition 1 (Directional field, vector field and direction field) A directional field denotes a function $\mathbf{v}$ that assigns directional information to almost every point in a given domain. If a directional field $\mathbf{v}$ provides a magnitude of importance for every point $p$, for which $\mathbf{v}$ is defined, then $\mathbf{v}$ is called a vector field (see Figure 2a). If the directional field does not provide any magnitude (e.g. all assigned vectors are normalized) it is called a direction field (see Figure 2b).

Definition 2 (N-directional field) A directional field can assign multiple directions to the same point in a domain $\Omega$. Let us assign $N$ vectors to almost every point in $\Omega$. Then the use-cases of $N=1,2,4$ are of importance to the application of the homogenization method. We write $\mathbf{N}$-vector or $\mathbf{N}$-direction field to indicate the number of directional information given at every point.

Figure 3 shows direction fields and vector fields important to the homogenization method, where we indicate a given magnitude with arrows. The 1-vector fields are what we usually refer to when talking about vector fields. If $N=2$ the vector is invariant under a rotation of angle $\pi$ and we call such a field a 2-vector field. For $N=4$ follows the 4 -vector field with a rotational symmetry of $\pi / 2$.

\subsection{Index of a singularity}

Singularities often arise in the presence of internal loads, multiple loads or a designated passive domain. The following definitions describe singularities and their indices. 


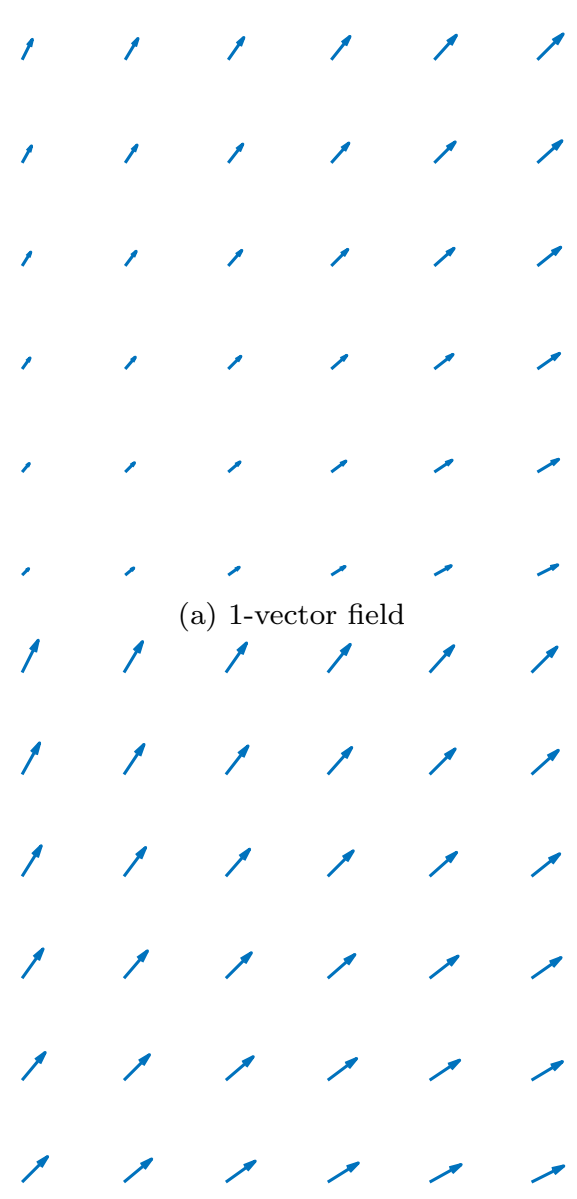

(b) 1-direction field

Fig. 2: Comparison of 1-vector and 1-direction field.

Definition 3 (Singular point, singularity) A point $\mathbf{p}$ in a 1-vector field $\mathbf{v}$ for which $\mathbf{v}$ is not defined or zero is called a singular point or singularity (see Figure 4).

Definition 4 (Index of a singularity) A singular point $\mathbf{p}$ can be assigned an index by the following procedure. We consider the vector field $\mathbf{v}$ on a closed, simple curve $\mathbf{c}(t), t \in[0,1]$, around $\mathbf{p}$, which does not contain a second singular point. We obtain $\mathbf{v}$ limited to $\mathbf{c}(\mathbf{t})$, by

$$
\mathbf{v}(\mathbf{c}(t))=\|\mathbf{v}(\mathbf{c}(t))\|\left(\begin{array}{c}
\cos (\alpha(t)) \\
\sin (\alpha(t))
\end{array}\right) .
$$

Here $\mathbf{c}:[0,1] \rightarrow \mathbb{R}^{2}$ denotes the counterclockwise parametrization of the curve and $\alpha:[0,1] \rightarrow \mathbb{R}$ the enclosed angle between the $\mathrm{x}$-axis and $\mathbf{v}(\mathbf{c}(t))$. The index of $\mathbf{p}$ is then defined as $\operatorname{idx}_{p}=\frac{1}{2 \pi}(\alpha(1)-\alpha(0))$ measuring the rotation of the vector field around the singular point.

In Figure 4 we consider a singularity in $(0,0)$ and depict the curve $c$ with $c(0)=c(1)=(1,0)$ as a red cir-
Vector fields Direction fields

1

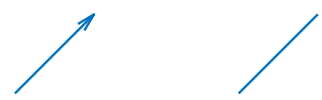

2

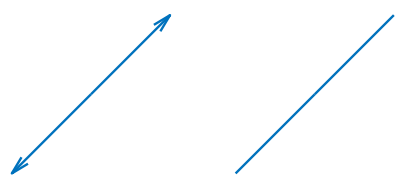

4
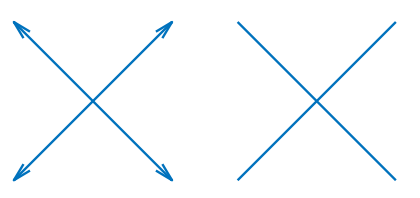

Fig. 3: Vector and direction fields for $N \in\{1,2,4\}$. A given magnitude is indicated by arrows.

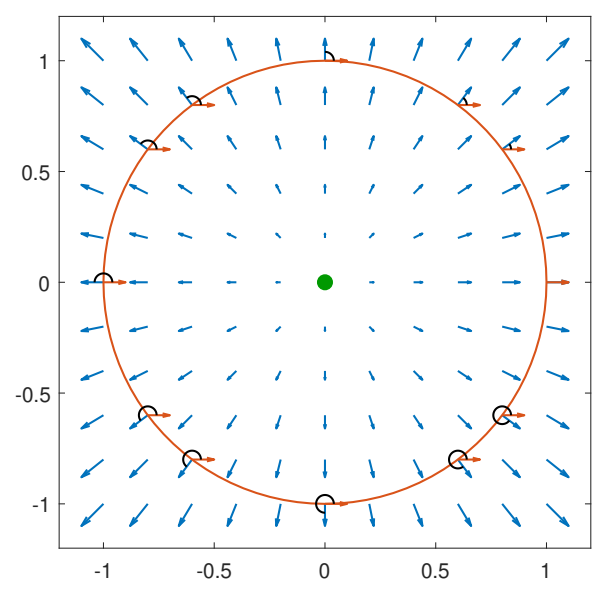

Fig. 4: Vector field containing a singularity of index 1 (in green), showing the tracked curve (in red) and the angle arcs (in black).

cle. Further, we see that the enclosed angles $\alpha$, depicted as black circle arcs, grow from $\alpha(0)=0$ to $\alpha(1)=2 \pi$. Thus we have a singularity (depicted in green) with in$\operatorname{dex}_{\operatorname{idx}}=\frac{1}{2 \pi}(2 \pi-0)=1$. Note that singularities exist for all types of $N$-directional fields, where the index must always be a multiple of $1 / N$. In order to identify the index of a singularity in a $N$-directional field, one proceeds similar to singularities in 1-directional fields. Following a closed curve around the singular point, one measures the change of the angle of the $N$-directional field. In a discrete example, as in Figure $7 \mathrm{~d}$ for example, one chooses one of the directions and follows that one along the curve. 


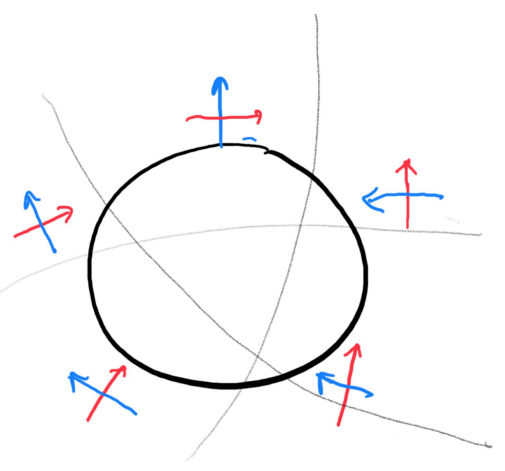

Fig. 5: Sketch of a singularity with index $1 / 4$.

\subsection{Singularities in smooth tensor fields}

The singularities of smooth tensor fields have been studied exhaustively in order to visualize tensor fields. Delmarcelle and Hesselink (1994) show that singularities in tensor fields can only have indices that are multiples of $\pm 1 / 2$. We try to present a descriptive version in the following theorem.

Theorem 1 A smooth tensor field can only encorporate singularities of index $\pm 1 / 2$ or multiples thereof.

Proof (Sketch) If we follow a closed, simple curve around a singularity $\mathbf{p}$, not containing a second singularity, with index $\pm 1 / 4$ as depicted in Figure 5. we see that red and blue vectors trade places. That would not be a problem for a 4-direction field where all four directions are equivalent, but if we have a tensor, we can distinguish the red and blue direction from each other by the magnitude of the eigenvalue. So, say blue is the direction of maximum eigenvalue that we follow, but coming back to the initial point it then must be red. The tensor has changed smoothly along the black curve, so there must have been a point along the black curve at which the eigenvalues were the same. However, if the eigenvalues are the same, any plane vector is an eigenvector, and we have a singular point. This contradicts the way the black curve was chosen and concludes the proof.

In homogenization-based topology optimization the layer normals are aligned with the stress tensors either by demanding it through the implementation or in a gradient descent algorithm since layers aligned to the load-path are optimal (Pedersen, 1989). Therefore, the following singularity types are found regularly in the output fields of the homogenization method.

For example, if all corners of a triangle are pulled apart (see Figure 6a) a singularity of index $-1 / 2$ occurs (see Figure 7a). Figure $7 \mathrm{~b}$ shows a singularity of type
-1 . This can be seen as two singularities of type $-1 / 2$ pushed together. This type of singularity occurs in a corner loaded square (see Figure 6b. Figure $7 \mathrm{c}$ shows a singularity of index $1 / 2$ as occurs for example in a center loaded clamped beam (see Figure6c).

\subsection{Singularities in 4-direction fields}

It is important to differentiate between the 4-vector field described by a stress tensor field and the 4direction field described by the layer normals output by the homogenization method. The latter can also incorporate singularities of index $\pm 1 / 4$ due to numerical errors, regularization of the layer-normals or simply due to the optimizer being stuck in a local minimum.

Figures $7 \mathrm{~d}$ and $7 \mathrm{e}$ show 4-direction fields incorporating each a singularity of index $\pm 1 / 4$. It is not possible to separate these 4-direction fields into two 1-direction fields, which is shown in Figure 7f. Especially regularization seems to result in singularities of index $\pm 1 / 4$. Increasing the regularization value $\gamma_{\theta}$ limits the angular changes. In Figures 8a- 8f we depict how a singularity of index $\pm 1 / 2$ is spatially ripped apart more and more with increasing regularization. In this example the singularity of index $\pm 1 / 2$ is split into two singularities of index $\pm 1 / 4$, that are located in the center of the domain at the end of the red lines. The red lines indicate where the vector fields jump by 90 or 180 degrees.

4.4 Occurrence of singularities in the homogenization results

For the de-homogenization, it is of importance to have an understanding of where singularities occur in the layer-fields output by the homogenization method. We are not aware of a theoretical solution to this, but we observe that, in general, singularities occur in regions with very high or very low density.

Let us assume, that in the output of the homogenization method, the layer-normals are well aligned with the stress directions everywhere, such that singularities in the stress field correspond to singularities in the layer-field. We investigate a singularity $\mathbf{p}$, where the stress field is non-zero. At such a singular point the stress field rotates with an index $\operatorname{idx}_{p} \neq 0$. Thus, in the immediate surrounding we see all directions in the interval $I=\left[0,2 \pi \mathrm{idx}_{p}\right]$. If we measure the angular velocity on several curves around $\mathbf{p}$, we realize that the shorter the curve, i.e. the closer to $\mathbf{p}$, the higher the angular velocity becomes, as the field always needs to rotate by $2 \pi \mathrm{idx}_{p}$. Or in other words, at $\mathbf{p}$ the stress field rotates infinitely fast. The homogenization method outputs two 


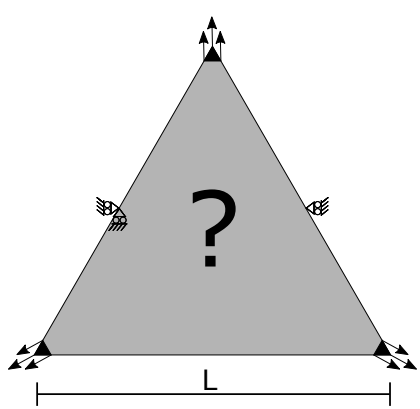

(a) Corner-loaded triangle

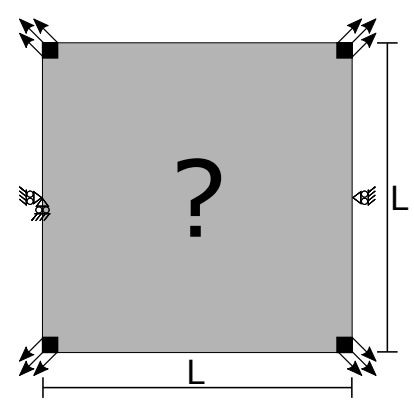

(b) Corner-loaded square

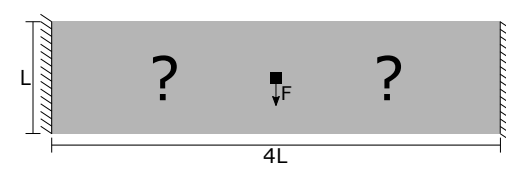

(c) Center-loaded clamped beam

Fig. 6: Examples of load cases leading to singularities.

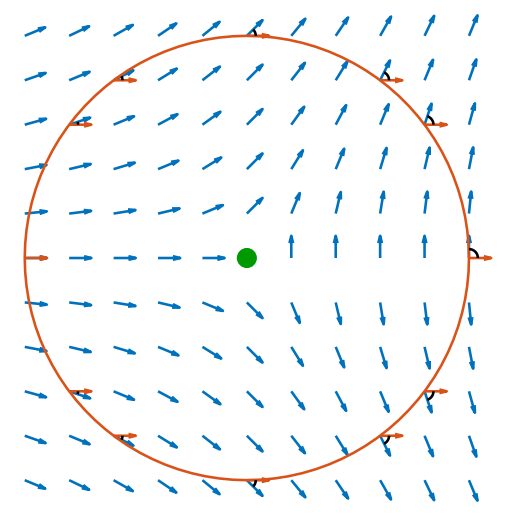

(a) 1 -vector field index $-1 / 2$

$+t+t+1+t+t$ $+t+1+t+t+x$ $+x+t+t+t+x$ $+x+t+t+t+x$ $x+t+t+x+x$ $+x+x+x \times x \times$ $\neq x+x \times \times \times \times \times \times x$ $x \times \times \times \times \times \times \times \times \times x$ $x \times x \times \times \times \times \times \times \times x$ $x \times \times x \times \times \times \times \times x$ $x \times \times \times \times \times \times \times \times x$

(d) 4-direction field index $1 / 4$

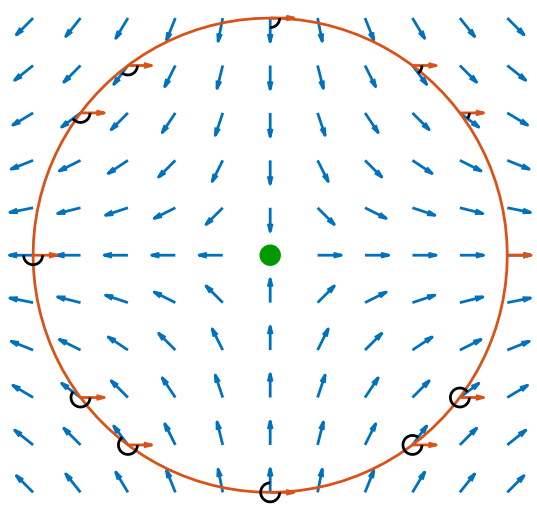

(b) 1-vector field index -1

$+t+t+1+t+t$ $+x+t+t+t+t$ $x+x+t+t+t+t$ $\mathrm{H}+\mathrm{x}+\mathrm{t}+\mathrm{t}+\mathrm{t}+\mathrm{H}$ $x+x+t+x+t$ *x $x \times x-4 x+x+x$ $x \times x \times x \times x \times x+$ $x \times x \times x \times x \times x \times x$ $x \times x \times x \times x \times x$ $x \times y \times x \times x \times x$ $x \times x \times x \times x \times x \times$

(e) 4-direction field index $-1 / 4$

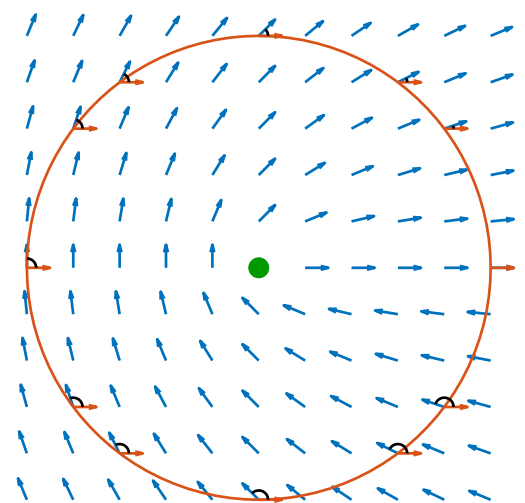

(c) 1-vector field index $1 / 2$

$1+111+11111$

$\begin{array}{lllllllllll}1 & 1 & 1 & 1 & 1 & 1 & 1 & 1 & 1\end{array}$

$\begin{array}{llllllllll}1 & 1 & 1 & 1 & 1 & 1 & 1 & 1 & 1\end{array}$

$\begin{array}{llllllllll}1 & 1 & 1 & 1 & 1 & 1 & 1 & 1\end{array}$

$\begin{array}{llllllllll}1 & 1 & 1 & 1 & 1 & 1 & 1 & 1\end{array}$

$1,1,1,11111$

$1,1,1,11111$

$1,1,1,11111$

$1,1,111111$

$1,1,1111111$

, 1 1 1 1 1 1 1 1

(f) 1-direction field index $-1 / 4$

Fig. 7: Vector and direction fields for different singularities.

layer-normals $\boldsymbol{n}^{1,2}$ and widths $w_{1,2}$ and thus a density $\rho=w_{1}+w_{2}-w_{1} w_{2}$ for the element containing $\mathbf{p}$. Now, if $0 \leq \rho<1$ only the directions $\boldsymbol{n}^{1,2}$ in $I$ are well aligned with the unit cell. Thus for all other directions in $I$ a shearing force will arise. The optimizer can improve the compliance by making the element containing $\mathbf{p}$ isotropic, which means that we can observe a density peak $\rho \rightarrow 1$ in the vicinity of the singularity $\mathbf{p}$. The same effect can be seen in Figure 8, where the material is distributed differently due to the layer directions differing from the stress direction due to smoothing. It is important to note that singularities also may occur in void regions. Following the above thought of using material to create isotropic elements, it is intuitively understandable that the optimizer mostly tries to stay clear of regions with fast turning stress fields and hence creates singularities in elements with $\rho=0$ aswell.

The above observation does not allow a prediction of where singularities occur in the layer-field. It does however allow to reason, why regions containing singularities can often be filled completely with material in the post processing without an excessive amount of 


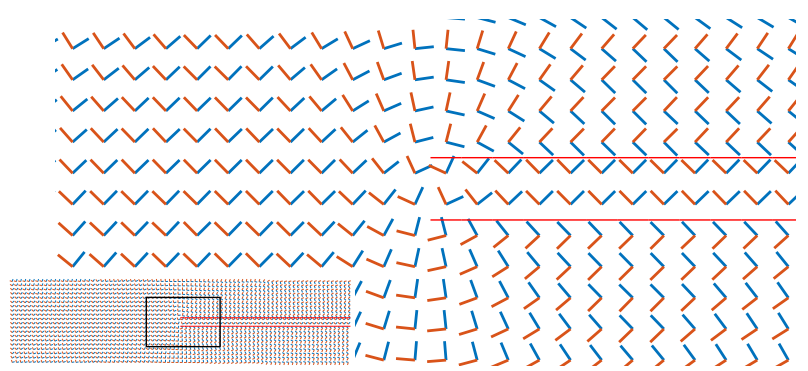

(a) Vector fields for $\gamma_{\theta}=0.0$

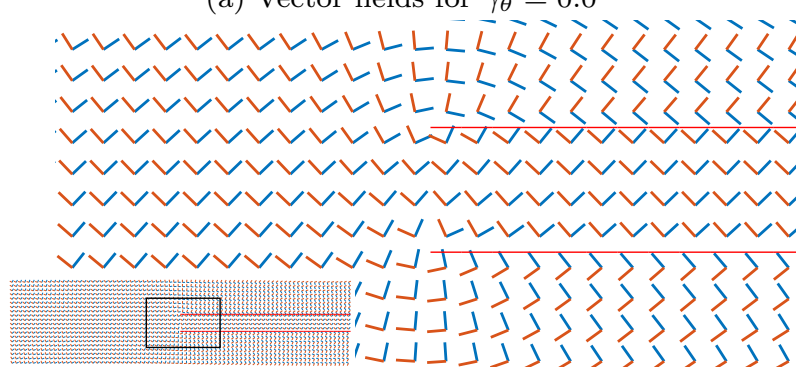

(c) Vector fields for $\gamma_{\theta}=0.1$

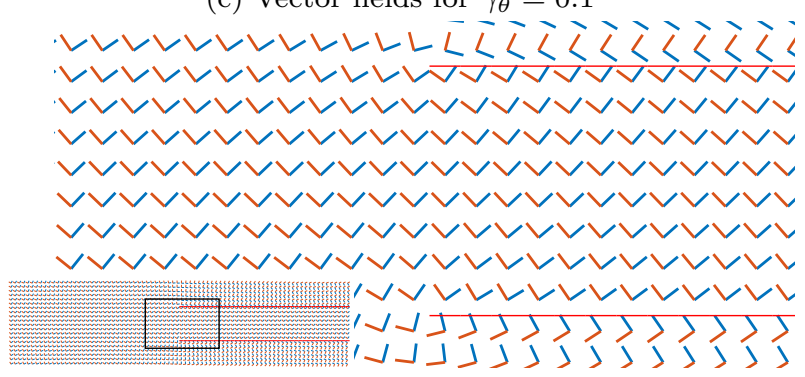

(e) Vector fields for $\gamma_{\theta}=0.5$

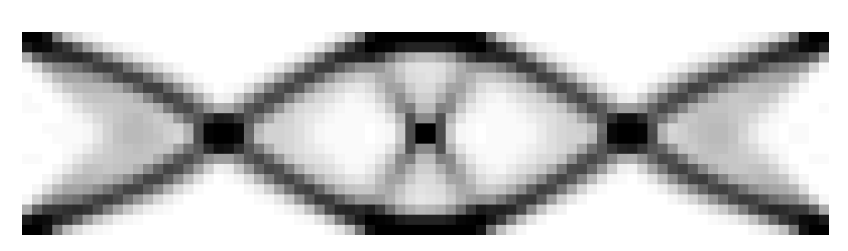

(b) Density for $\gamma_{\theta}=0.0$

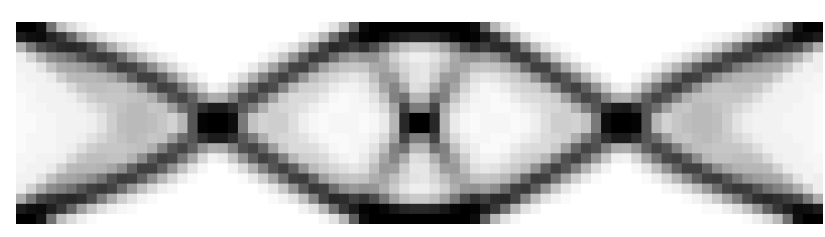

(d) Density for $\gamma_{\theta}=0.1$

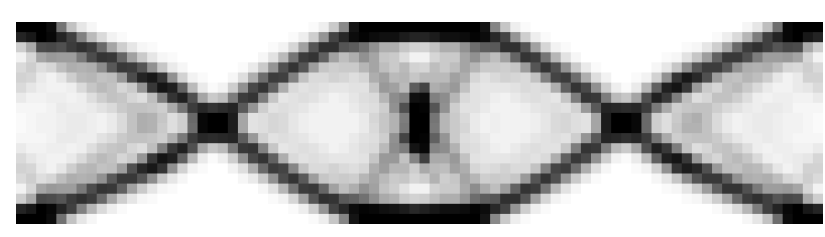

(f) Density for $\gamma_{\theta}=0.5$

Fig. 8: Vector fields and densities for different degrees of regularization.

additional material needed as we already see a density peak. One needs to be careful as the above observation assumes layer-fields that are well aligned to the principal stress directions.

\section{De-homogenization for singularity containing 4-direction fields}

With the knowledge accrued about singularities in the last section, we are now able to study examples that contain singularities. In this section we revisit approaches for the de-homogenization in singularity free problems (see Subsection 5.1), propagate consistent labeling of the direction fields in the case of singularities (see Subsection 5.2 and show the connection to quadmeshing (see Subsection 5.3.
5.1 De-homogenization for smooth, continuous and singularity free 4-direction fields

Similar to Pantz and Trabelsi (2008); Groen and Sigmund (2018); Allaire et al. (2018) we de-homogenize the square unit-cell with a rectangular hole using two periodic layers superimposed onto each other. Provided that vector fields are smooth we can use the procedure as presented in Groen et al. (2019) which will be repeated here for convenience.

Based on the two smooth 1-direction fields $\boldsymbol{n}^{i}, i \in$ $\{1,2\}$ we create two mapping functions $\phi_{1}$ and $\phi_{2}$ that preserve the orientation of the microstructures. Nevertheless, it should be noted that the map is not strictly conformal. The values of $\phi_{i}$ do not need to be accurate when there is no material in the layer (i.e. $w_{i}<0.01$ ) or when the domain is completely solid (i.e. $\rho>0.99$ ). Hence, the problem only needs to be solved accurately in the intermediate material part of the domain of the $i-t h$ layer $\tilde{\Omega}_{i}$. This leads to the following spatially 
weighted partial differential equations (PDE),

$$
\begin{array}{rrr} 
& \alpha_{1}^{i}(\boldsymbol{x}) \nabla \phi_{i}(\boldsymbol{x})=\alpha_{1}^{i}(\boldsymbol{x}) \boldsymbol{n}^{i}(\boldsymbol{x}), \quad \boldsymbol{x} \in \Omega \\
\text { s.t. } & \alpha_{2}^{i}(\boldsymbol{x}) \nabla \phi_{i}(\boldsymbol{x}) \cdot \boldsymbol{t}^{i}(\boldsymbol{x})=0, \quad \boldsymbol{x} \in \Omega
\end{array}
$$

where $\boldsymbol{t}^{i}$ denotes the orthogonal vector to $\boldsymbol{n}^{i}$. The domain is split up into three parts, which dictate the weights on the objective $\alpha_{1}^{i}$ and the weights on the constraints $\alpha_{2}^{i}$,

$\alpha_{1}^{i}(\boldsymbol{x})=\left\{\begin{array}{lll}0.01 & \text { if } & w_{i}(\boldsymbol{x})<0.01, \\ 0.1 & \text { if } & \rho(\boldsymbol{x})>0.99, \\ 1 & \text { if } & \boldsymbol{x} \in \tilde{\Omega}_{i} .\end{array}\right.$

$\alpha_{2}^{i}(\boldsymbol{x})=\left\{\begin{array}{lll}0 & \text { if } & w_{i}(\boldsymbol{x})<0.01, \\ 0 & \text { if } \quad \rho(\boldsymbol{x})>0.99, \\ 1 & \text { if } \quad \boldsymbol{x} \in \tilde{\Omega}_{i} .\end{array}\right.$

Numerically, we solve the above-mentioned problem as a linear system using the finite element approach, where the constraint is enforced in an augmented setting using penalty parameter $\gamma_{\phi}$, called the alignment weight. With both mapping fields known we can create an implicit geometry description $\tilde{\rho}_{i}$ for each of the layers:

$\tilde{\rho}_{i}(\boldsymbol{x})=H\left(\left(\frac{1}{2}+\frac{1}{2} \mathcal{S}\left\{P_{i} \phi_{i}(\boldsymbol{x})\right\}\right)-\tilde{w}_{i}(\boldsymbol{x})\right)$.

Here $H$ is the Heaviside function and $\mathcal{S} \in[-1,1]$ corresponds to a triangle wave. Furthermore, $P_{i}$ is a periodicity scaling. Hence, the design can be de-homogenized by an implicit geometry function $\tilde{\rho}$ as,

$\tilde{\rho}(\boldsymbol{x})=\min \left\{1, \sum_{i=1}^{2} \tilde{\rho}_{i}(\boldsymbol{x})\right\}$.

Finally, we can impose an average layer spacing $\varepsilon$, which can be interpreted as the unit-cell size. To do so, we define the periodicity scaling parameter $P_{i}$ based on the average lattice spacing in the domain of interest $\tilde{\Omega}_{i}$,

$P_{i}=\frac{2 \pi}{\varepsilon} \frac{\int_{\tilde{\Omega}_{i}} \mathrm{~d} \tilde{\Omega}_{i}}{\int_{\tilde{\Omega}_{i}}\left\|\nabla \phi_{i}(\boldsymbol{x})\right\| \mathrm{d} \tilde{\Omega}_{i}}$,

where the average layer spacing $\varepsilon$ allows to steer the average spacing between the structural members.

We depict the main steps in Figure 9 From a 4direction field (Figure 9a) two 1-direction fields are extracted (Figure 9b) for a standard cantilever beam. The corresponding parametrization $\phi_{i}$ is depicted in Figure $9 \mathrm{c}$, where contour lines have been added for a better understanding. Figure $9 \mathrm{~d}$ shows the cosine of the parametrization indicating all isocontours and the periodic structure. Figure $9 \mathrm{e}$ shows $\tilde{\rho}_{i}$ and ultimately $\tilde{\rho}$ is depicted in Figure 9f. For further examples of dehomogenization using smooth 1-direction fields in 2and $3 \mathrm{D}$, the reader is referred to Groen and Sigmund (2018); Groen et al. (2020, 2019).

\subsection{De-homogenization in the presence of singularities}

In the presence of singularities the above described methods can fail for several reasons. Depending on the index of the singularity different issues can arise:

- If a singularity of index \pm 1 is contained in the 4direction layer-normal field, the separation into two integrable 1-direction fields is still possible (see Figure $7 \mathrm{~b}$. As locally neighboring vectors need to be pointing in the same direction the extraction of vector-fields does not arise trivially. This extraction is called combing. In practice, a breadth-first search has shown to fail with this task as depicted in Figure $10 \mathrm{~b}$ because the singular point is hit. More robust results can be reached by expanding the search through intermediate densities first, before expanding into void and solid. This algorithm has been used in 3D in Groen et al. (2020) and is explained in Subsection 5.2.1 and depicted in Figure 11 and the result for the corner loaded square in $10 \mathrm{c}$.

- In the case of a singularity of index $\pm 1 / 2$ the 4 direction field can still be combed, such that two separate 1-direction fields arise (see Figure $7 \mathrm{a}, 7 \mathrm{c}$ ). However, the combing of these 1-direction fields cannot be done consistently anymore. Any combing strategy will inevitably create mismatches, i.e. some neighboring elements will contain vectors that are rotated by $\pi$ (see further Subsection 5.2.2). A promising approach to extract a high-resolution structure for such fields has been proposed in Donders (2018).

- In case of a singularity with index $\pm^{1 / 4}$ the whole procedure gets even more challenging. Recall that following a curve $c$ around a singularity of index $1 / 4$ in a 4-direction field means that we return to the start of our curve misaligned by $\pi / 2$ (see Figure $7 \mathrm{~d}, 7 \mathrm{f}$ ). Therefore, we cannot find two 1-direction fields to which we can assign the directions of our 4-direction field. Instead one needs to cut open the field in such a way, that one can integrate the 1direction fields using multiple coverings as introduced in Kälberer et al. (2007). We will discuss this topic extensively in Subsection 5.3 .

\subsubsection{Combing the 4-direction field}

As a first step, we have to comb our 4-direction field. This means we want to choose two orthogonal 1- 


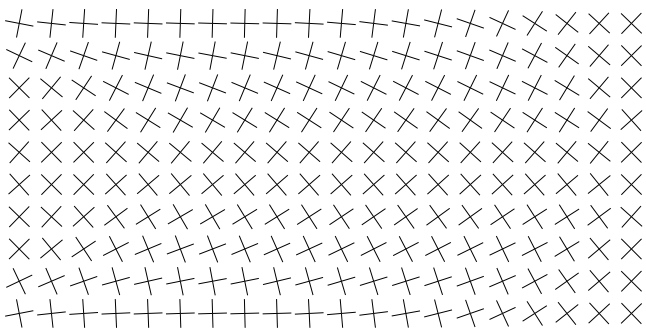

(a) 4-direction field

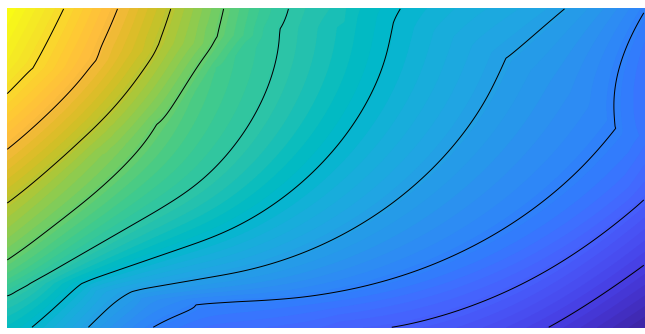

(c) Parametrization with some isocurves

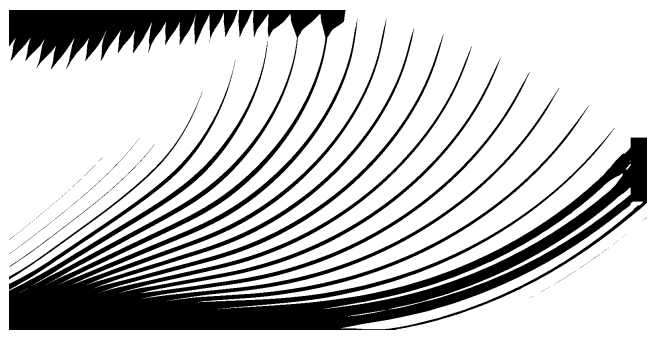

(e) Projection using $\omega$

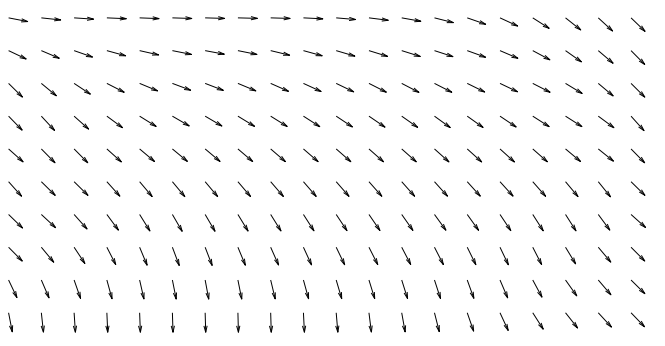

(b) 1-direction field

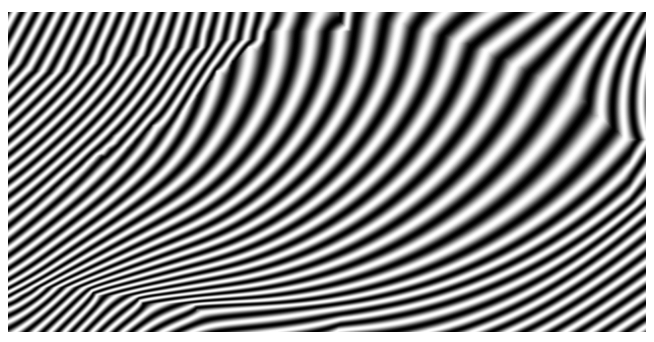

(d) Cosine wave

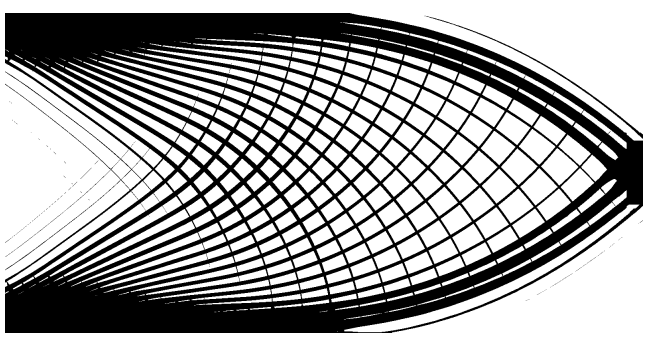

(f) Combining two layer projections

Fig. 9: Projection pipeline - from a 4-direction field to de-homogenized mechanical structure.

directions for every element. In quad meshing, this is normally done with a breadth-first search as shown in Kälberer et al. (2007). As the homogenization method results are noisy and unreliable in normals of layers with very low or very high width, it makes sense to prioritize combing in medium-density regions. This has recently been proposed in Groen et al. (2020) and we depict an example of our implementation in Figure 11, where the chosen directions are given as black arrows and seam edges are depicted in bright red. We color the elements according to the time where they are visited by the combing algorithm. For readability we bundle 50 elements to one step and color them with the same color. Unlike Groen et al. (2020) we only use the direction of a single neighboring element to compute the direction to be fixed in element $f$. This allows for a much faster algorithm and is possible due to the angular informa- tion being more robust in $2 \mathrm{D}$. The density priority is obtained by $\rho_{\text {priority }}=\left|\rho_{\text {start }}-\rho_{f}\right|$, where normally $\rho_{\text {start }}=0.5$ is chosen. We therefore comb the field first in areas with a density close to 0.5 and then spread out into the remaining domain. The numerical implementation of the combing only selects one direction per element. The second direction is implicitly given as the rotation of the extracted 1-direction field by $\pi / 2$.

\subsubsection{Finding the seams}

For every 4-direction field containing a singularity with index $1 / 4$ or $1 / 2$, we receive a combed 1 -direction field that has jumps of angle $\pi / 2$ and/or $\pi$. Such fields are not integrable and to be able to parameterize our domain, we need to cut open our mesh as done in Kälberer et al. (2007). 
Definition 5 (Seams, mismatch, seam edge) Two directions whose enclosed angle exceeds $\pi / 4$ are said to mismatch by $\pi / 2$. Accordingly, two directions whose enclosed angle exceeds $3 \pi / 4$ are said to mismatch by $\pi$. An edge $e$ is called seam edge if the directions in the two adjacent elements $f_{1}, f_{2}$ mismatch. A seam is any series of connected seam edges.

\subsection{Constrained mixed integer solver}

In Quad-meshing, multiple coverings and thus resulting seams were introduced in Kälberer et al. (2007). An expansion introducing an iterative scheme to enforce integers is given in Bommes et al. (2009). We want to exploit the underlying mechanisms to create a topology on which we can integrate the combed fields, combining them with the integration scheme introduced in Groen and Sigmund (2018). Therefore, we give a short simplified introduction to Bommes et al. (2009) in Subsections 5.3 .15 .3 .3 .

\subsubsection{Cutting the mesh open}

As a first step, one cuts open the mesh along the seams created in Subsection 5.2.2 Let $e=\left(v_{1}, v_{2}\right)$ be a seam edge, connecting the vertices $v_{1}$ and $v_{2}$ as in Figure 12. The edge $e$ separates the two elements $f_{1}$ and $f_{2}$. Auxiliary vertices $\tilde{v}_{1}$ and $\tilde{v}_{2}$ are introduced and the connectivity of $f_{2}$ is changed from $\left[v_{1}, v_{2}, v_{t}, v_{s}\right]$ to $\left[\tilde{v}_{1}, \tilde{v}_{2}, v_{t}, v_{s}\right]$.

\subsubsection{Gluing the mesh together}

If one would solve the resulting linear system in Equation 6 on this cut open mesh, disconnected structural members would arise at the seam locations, since the integer isolines on the left and the right side of the seam would not meet up. Therefore, one needs to make sure that the parametrizations $\phi_{1}$ and $\phi_{2}$ in vertex $v$ differ by integers $i, j$ from the parametrizations in $\tilde{v}$. This ensures connected bars and also the same spacing between bars on both sides of the seam. In case of a singularity of $1 / 4$ the parametrization $\phi_{1}$ can corresponds to the parametrization $\phi_{2}$ on the other side. These rotations $\operatorname{Rot}_{90}^{e}$ are given implicitly by the combed field. Kälberer et al. (2007) formulate the rotational and integer condition with the following equations

$$
\begin{aligned}
& \left(\phi_{1}\left(\tilde{v_{1}}\right), \phi_{2}\left(\tilde{v_{1}}\right)\right)=\operatorname{Rot}_{90}^{e}\left(\left(\phi_{1}\left(v_{1}\right), \phi_{2}\left(v_{1}\right)\right)\right)+(i, j), \\
& \left(\phi_{1}\left(\tilde{v_{2}}\right), \phi_{2}\left(\tilde{v_{2}}\right)\right)=\operatorname{Rot}_{90}^{e}\left(\left(\phi_{1}\left(v_{2}\right), \phi_{2}\left(v_{2}\right)\right)\right)+(i, j),
\end{aligned}
$$

where $i, j \in \mathbb{Z}$ enforces that the integer isolines meet up. The operator $\operatorname{Rot}_{90}^{e}$ accounts for the mismatch of the rotations on both sides of the seam. It can be seen as a multiplication with the imaginary number $i$ in the complex plane, where we regard the parameterizations $\left(\phi_{1}\left(v_{1}\right), \phi_{2}\left(v_{1}\right)\right)$ as a complex number. Maybe most intuitively is the rotation with angle $\pi$. When we look at Figure 12 and think of the vectors as gradients of the parameterizations $\phi_{1}$ and $\phi_{2}$, we see, that if the value of a parameterizations $\phi$ increases on one element, then it must be decreasing on the other element. Thus the signs of the parameterizations need to change, which corresponds to a multiplication with $i^{2}$. For rotations of angle $\pi / 2$ or $3 \pi / 2$ the two parameterizations $\phi_{1}$ and $\phi_{2}$ need to switch position, since the "blue" field becomes the "red" field. This corresponds to a multiplication with $i$ or $i^{3}$ in the complex plane. If we define the angle as the rotation in counterclockwise direction, we get:

$\operatorname{Rot}_{90}^{e}\left(\left(\phi_{1}\left(v_{1}\right), \phi_{2}\left(v_{1}\right)\right)\right)=i^{l(e)}\left(\phi_{1}\left(v_{1}\right), \phi_{2}\left(v_{1}\right)\right)$,

where $l(e) \in\{1,2,3\}$ is given by the mismatch of the angles at edge $\mathrm{e}$.

Further details about the operator $\operatorname{Rot}_{90}^{e}$ and this procedure can be found in Kälberer et al. (2007); Bommes et al. (2009).

\subsubsection{Solving for the parametrization}

Once the system is assembled, it is solved in the continuous case, i.e. no integer restrictions are applied at first. This yields an approximation to the desired solution solving the problem up to disconnected bars at the seams. The mismatch variables $(i, j)$ can now be seen as slack variables $j \in J$, where $J$ is the set of all slack variables. For every iteration the slack variable closest to an integer

$$
k=\underset{j \in J}{\arg \min }|j-\operatorname{round}(j)|,
$$

is enforced to round $(k)$ and the new system is solved. In an iterative fashion, this procedure is continued until no more slack variables are left.

\section{Parameter choice for homogenization and de-homogenization}

The homogenization and the de-homogenization are both influenced by some key parameters. The pipeline introduced in Section 5 allows us to study different examples of start orientations for the layer normal and regularization values (see Subsection 6.1). Further we also outline the important de-homogenization parameters (see Subsection 6.2). 


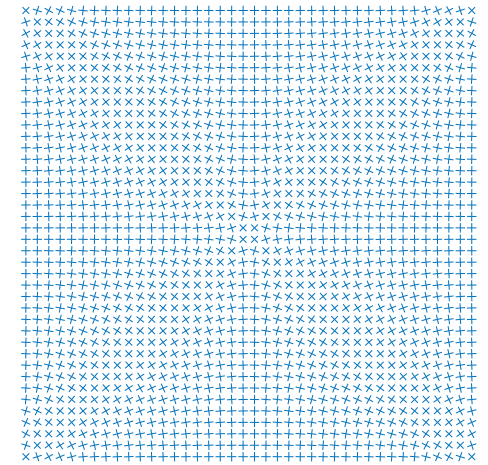

(a) 4-direction field before combing

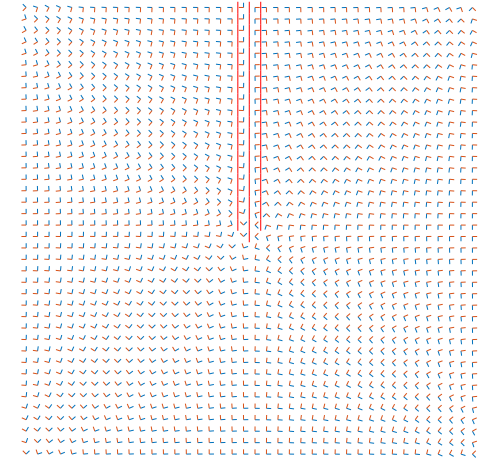

(b) Breadth-first combing

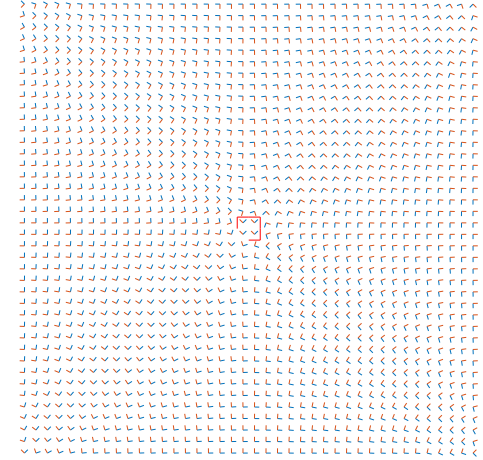

(c) Density-based combing

Fig. 10: Example of two different combing strategies for a given 4-direction field resulting in different seams. The breadth-first combing only uses information about the neighborhood of elements, whereas the density-based combing uses density values to prioritize the combing of elements.

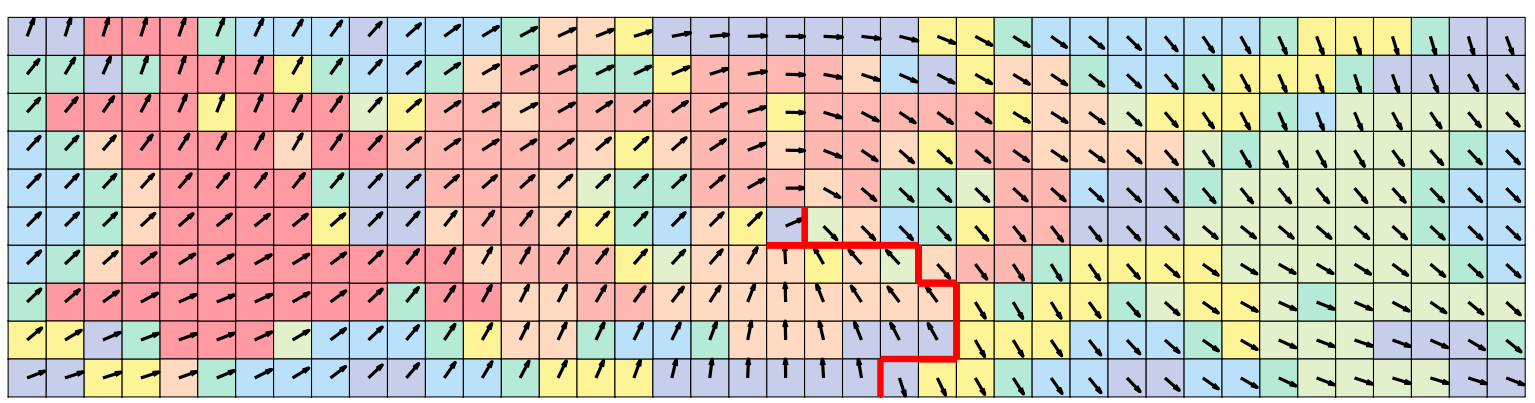

Fig. 11: Extraction of a 1-direction field using a density-based combing strategy. The combing is started at a root element and follows intermediate densities first. The colors show steps of 50 elements at a time, whose 1-direction has been fixed. The colors show steps of 50 elements at a time, whose 1-direction has been fixed.
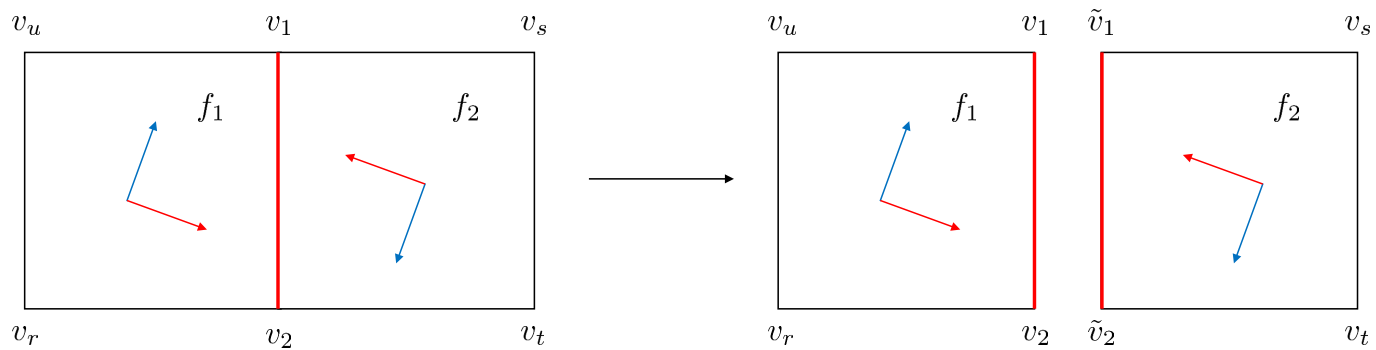

Fig. 12: Sketch of cutting a grid open between two neighboring elements at a seam edge.

6.1 Influence of the start point choice and the regularization of the homogenization method

It is known for the corner loaded square (see Figures 6b. 13 - 16 that there does not exist a unique optimal solution. The shown examples were all achieved using a resolution of $120 \times 120$ elements for the topology optimization as well as for the parametrization step. To visualize the resulting structures, we choose for the de-homogenization an average layer spacing $\varepsilon=5 h_{f}$, where $h_{f}=15$ is the number of fine scale elements used, and an alignment weight $\gamma_{\phi}=50$. For the examples in Figures 14 and 16 the value $\gamma_{\theta}=\frac{1}{5}$ is applied. In this example we see that the start guess dictates the resulting structure. If the start point for the homogenization method consists of a grid aligned 4-direction field we receive the structures depicted in Figures 15 and 16 that are fairly grid aligned. However, if we solve one finite element step, assuming that in all elements we have a density $\rho=1$, we receive a stress tensor field, whose eigenvectors can be used as a start guess for the homogenization method, as shown in Groen et al. (2020). 


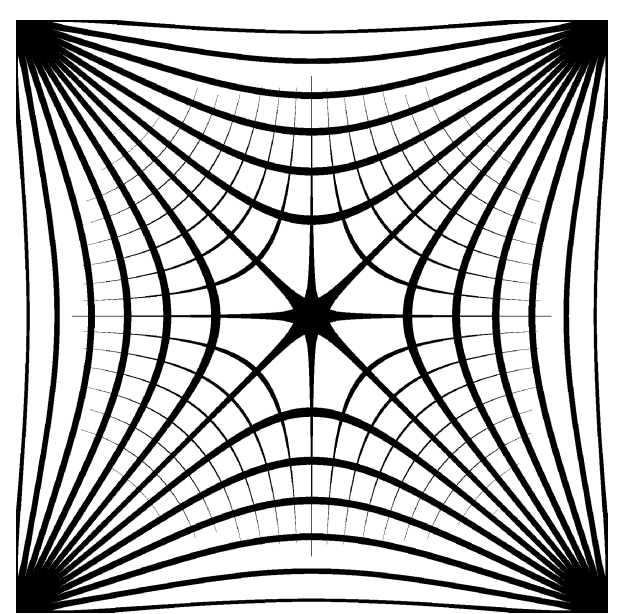

(a) Structure

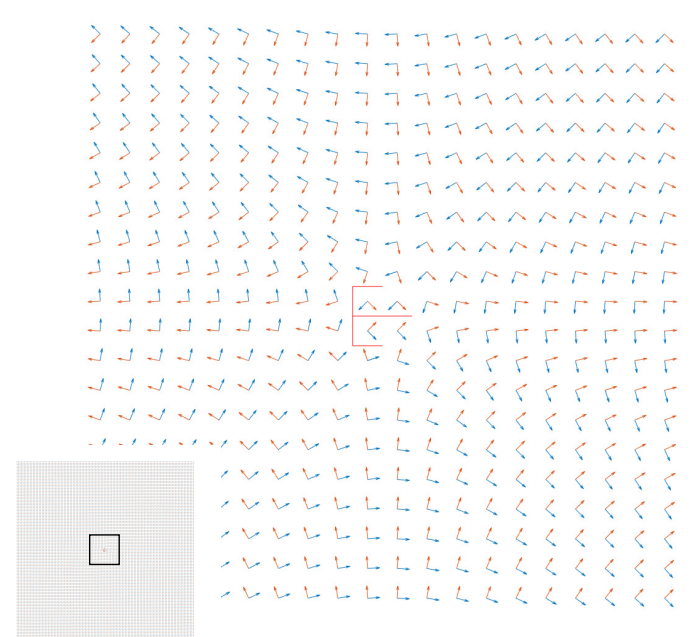

(b) Enlargement of the centre of the combed vector fields

Fig. 13: Example of a corner loaded square using principal stresses as a start guess for the layer-orientation, no regularization applied.

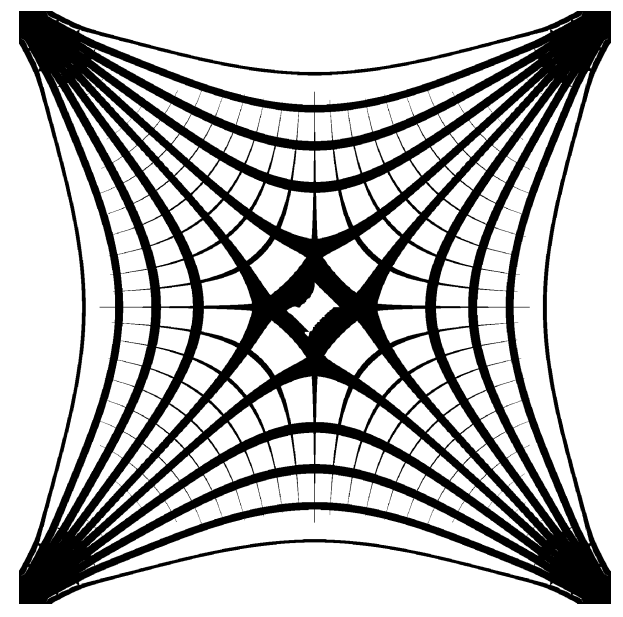

(a) Structure

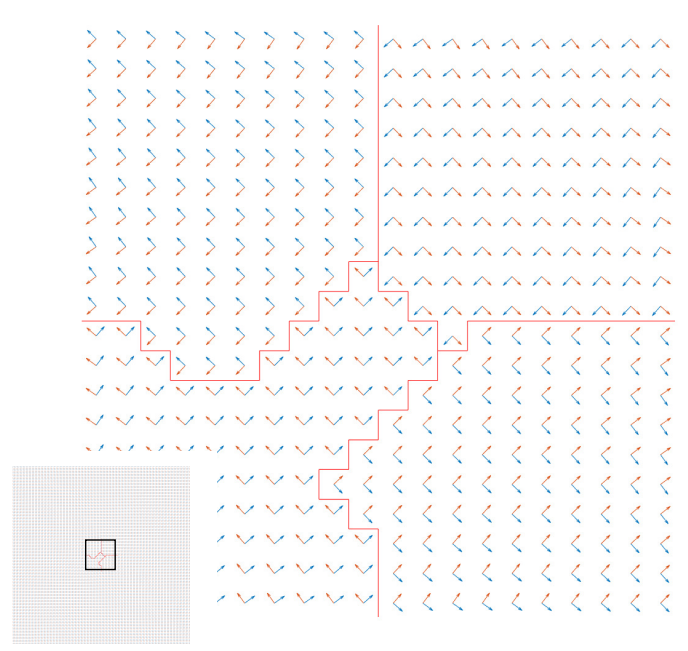

(b) Enlargement of the centre of the combed vector fields

Fig. 14: Example of a corner loaded square using principal stresses as a start guess for the layer-orientation, regularization $\gamma_{\theta}=\frac{1}{5}$ applied.

The results differ from the grid aligned start guess and are shown in Figures 13 and 14 . Note that even if we impose regularization, the start guess still dictates the outcoming structure as shown in the Figures 14 and 16 . With the principal stress directions as starting guess we receive spatially ripped apart singularities, when imposing regularization. With the uniform grid as starting guess we stay completely clear of getting a singularity in the center of the domain. Note that despite the large geometric variations in these solutions, compliance values $C_{h}$ for obtained homogenization results are extremely close demonstrating the non-uniqueness of this problem. 


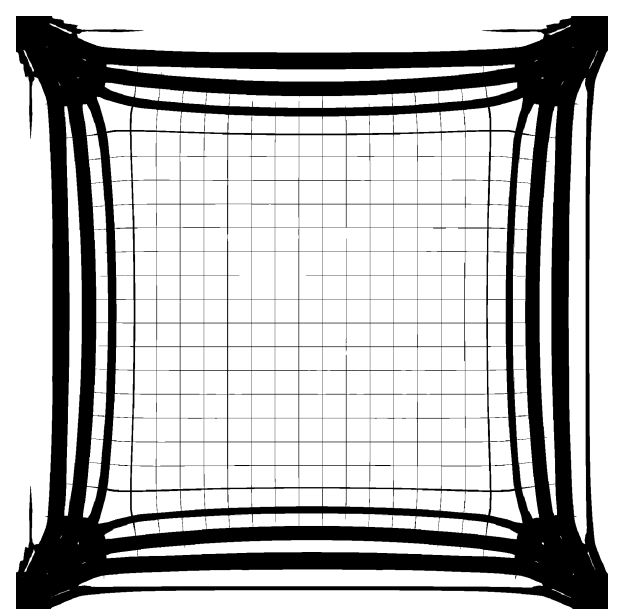

(a) Structure

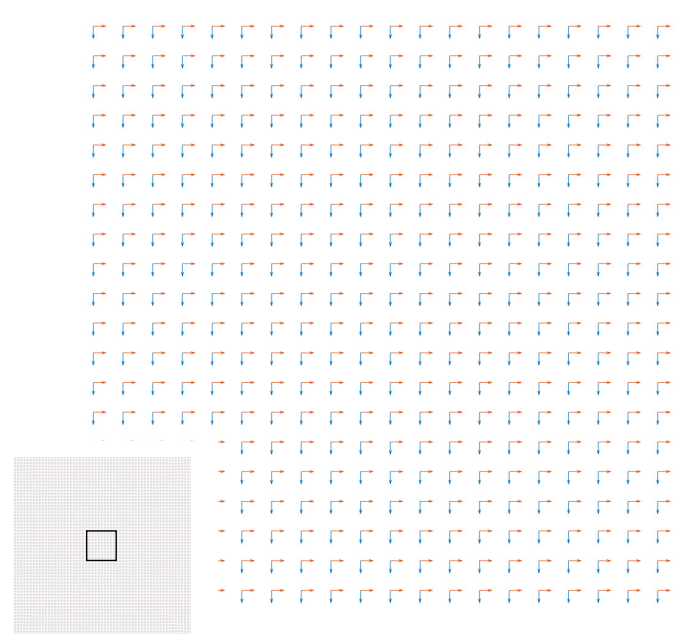

(b) Enlargement of the centre of the combed vector fields

Fig. 15: Example of a corner loaded square using grid aligned directions as a start guess for the layer-orientation, no regularization applied.

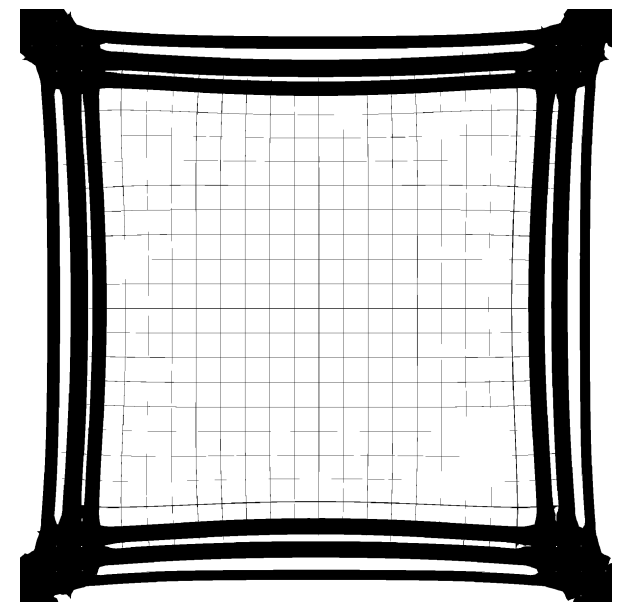

(a) Structure

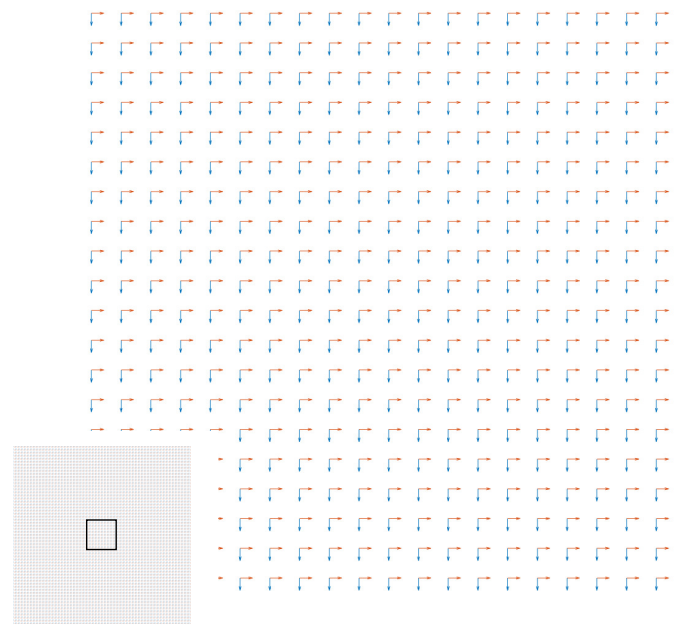

(b) Enlargement of the centre of the combed vector fields

Fig. 16: Example of a corner loaded square using grid aligned directions as a start guess for the layer-orientation, regularization $\gamma_{\theta}=\frac{1}{5}$ applied.

6.2 Alignment weight $\gamma_{\phi}$ and structural member thickness $\varepsilon$

There are two main parameter choices influencing the result during the de-homogenization: The alignment weight $\gamma_{\phi}$ and the average layer spacing $\varepsilon$. We refer to Groen and Sigmund (2018) for the choice of the layer spacing $\varepsilon$. For comparison reasons, we use in this subsection an $\varepsilon=10 / L$, where $L$ is the resolution in vertical direction. Further, topology optimization and de-homogenization are done on the same resolution. If no singularity is present, then the alignment weight $\gamma_{\phi}$ should be chosen in a range of 100-1000 as shown in Groen et al. (2019). It holds, that the lower the maximal absolute divergence of a vector field, the bigger $\varepsilon$ can be chosen, as it simply enforces a very strict alignment to the second vector field. However, in case of a singular point, the field spins with a certain index 
around that point. If we now enforce a big alignment weight on a low-resolution ( $80 \times 20$ elements) example we receive stretched isocontours as shown in Figure 17a. The gradient of the parametrization becomes almost zero in a large region. The constraint of Equation 6 is implemented as a penalization term multiplied with the alignment weight $\gamma_{\phi}$. Note that this term can be minimized by the gradient becoming zero due to the usage of the dot product. However, this leads to an increase in the first term of Equation 6 and an unwanted stretching of the isocontours. For a low-resolution example, the space of bi-linear basis-functions is limited and thus the parametrization is limited in adapting to the rotation around a singularity. This leads to smearing out of the rotational influence from the singularity to neighboring elements. If the element size is large, this influences a higher relative area of the optimization domain. Thus if the alignment weight is chosen very high the gradient becomes zero in these elements due to the first term of Equation 6 being suboptimal already. This often results in a violation of the volume constraint as can be extracted from Table 1 In order to allow for a better comparison we provide the value $V_{s} C_{s}$, which should however not be considered as a sole objective, since the compliance does not depend linearly on the volume. By using a higher resolution $(160 \mathrm{x} 40,320 \mathrm{x}$ $80,640 \times 160$ ) (see Figures $17 \mathrm{~b}, 17 \mathrm{c}$ and Table 1) most of this problem is resolved. We can see that a trade off in the alignment weight is still desirable for these highresolutions. On the one hand, a low alignment weight can lead to non-load bearing bars, as isocurves might lead into an area with zero layer width due to bad alignment. On the other hand, a too large alignment weight can lead to an overvaluing of the constraint and thus to stretched isocontours. For the examples in Table 1. we observe that the optimal alignment weight can depend on the resolution $\gamma_{\phi}$ between 50 and 5000 dependent on the de-homogenization grid.

\subsection{Island removal via connected component}

To get rid of elements that are neither connected to the boundary condition nor to the load, we do a simple connected component analysis, where we only retain the largest component. This immediately leads to the desired removal of islands. However this still leaves some unloaded bars, these can be removed by a couple of FE analysis and removal of unloaded elements as proposed in Groen and Sigmund (2018).

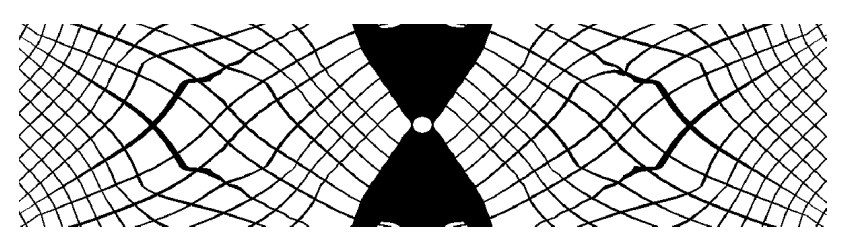

(a) Isocontours with resolution $80 \times 20$ and alignment weight $\gamma_{\phi}=500$

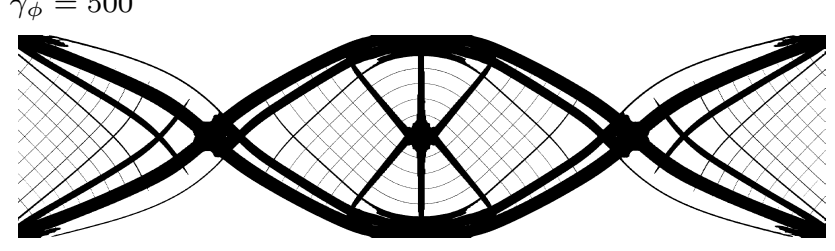

(b) Structure with resolution $320 \times 80$ and alignment weight $\gamma_{\phi}=5000$

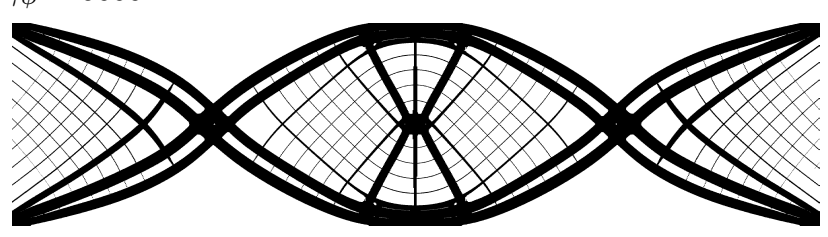

(c) Structure with resolution $640 \times 160$ and alignment weight $\gamma_{\phi}=5000$

Fig. 17: Examples of center loaded clamped beam for different resolutions and alignment weights.

Table 1: Performance for different alignment weights $\gamma_{\phi}$ and resolutions res. We use the following abbreviations: $C_{h}=$ compliance of homogenization method, $V_{s}, C_{s}=$ volume and compliance of de-homogenized structure, $t_{h}, t_{s}, t=$ time for homogenization-based topology optimization, the dehomogenization and the overall time in seconds. Since the compliance does not depend linearly on the volume $V_{s} C_{s}$ should not be considered as the sole objective.

\begin{tabular}{|c|c|c|c|c|c|c|c|c|}
\hline res & $\gamma_{\phi}$ & $C_{h}$ & $V_{s}$ & $C_{s}$ & $V_{s} C_{s}$ & $t_{h}$ & $t_{s}$ & $t$ \\
\hline \multirow{4}{*}{$\begin{array}{l}\stackrel{\sim}{N} \\
\ddot{x} \\
\stackrel{\infty}{\infty}\end{array}$} & 0 & 21.89 & 0.298 & 61.79 & 18.42 & 44.8 & 5.4 & 50.2 \\
\hline & 50 & 21.89 & 0.316 & 32.60 & 10.30 & 44.8 & 6.2 & 51.0 \\
\hline & 500 & 21.89 & 0.349 & 24.26 & 8.45 & 44.8 & 5.2 & 50.0 \\
\hline & 5000 & 21.89 & 0.351 & 24.50 & 8.60 & 44.8 & 4.0 & 48.7 \\
\hline \multirow{4}{*}{$\begin{array}{l}\stackrel{ }{+} \\
x \\
\stackrel{0}{0} \\
\stackrel{-}{1}\end{array}$} & 0 & 21.82 & 0.295 & 337.74 & 99.52 & 135.4 & 11.2 & 146.6 \\
\hline & 50 & 21.82 & 0.334 & 24.69 & 8.25 & 135.4 & 10.2 & 145.6 \\
\hline & 500 & 21.82 & 0.343 & 22.76 & 7.81 & 135.4 & 10.2 & 145.7 \\
\hline & 5000 & 21.82 & 0.338 & 23.46 & 7.93 & 135.4 & 10.8 & 146.2 \\
\hline \multirow{4}{*}{ 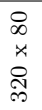 } & 0 & 21.84 & 0.297 & 71.95 & 21.39 & 402.9 & 34.3 & 437.2 \\
\hline & 50 & 21.84 & 0.313 & 22.52 & 7.05 & 402.9 & 30.8 & 433.8 \\
\hline & 500 & 21.84 & 0.300 & 24.89 & 7.48 & 402.9 & 30.7 & 433.7 \\
\hline & 5000 & 21.84 & 0.285 & 26.75 & 7.63 & 402.9 & 30.8 & 433.7 \\
\hline \multirow{4}{*}{$\begin{array}{l}\stackrel{8}{0} \\
\ddot{1} \\
\ddot{0} \\
\stackrel{+}{0}\end{array}$} & 0 & 22.02 & 0.298 & 73.51 & 21.90 & 1824.5 & 112.8 & 1937.3 \\
\hline & 50 & 22.02 & 0.293 & 25.57 & 7.48 & 1824.5 & 109.9 & 1934.3 \\
\hline & 500 & 22.02 & 0.288 & 25.91 & 7.46 & 1824.5 & 147.1 & 1971.6 \\
\hline & 5000 & 22.02 & 0.313 & 22.07 & 6.91 & 1824.5 & 111.5 & 1936.0 \\
\hline
\end{tabular}

\section{Conclusion and future work}

We have identified the singularities occurring most often in 4-direction fields arising from homogenization based topology optimization. Further, we investigated the location and reasons for singularities to occur. We have shown the influence of the start guess of the layerorientations on the outcome of the optimization. The 
proposed parametrization approach using Quad-Cover allows parametrizing fields containing singularities of index $\pm 1 / 4$. The proposed parametrization approach also allows us to cut out areas completely filled with void or solid material from the parametrization step. These contributions increase the number of designs for which the de-homogenization method can generate high-resolution near-optimal structures at a low computational cost. In order for the aforementioned points to come to carry the next step for further research is the adaptation of the projection step outlined in Groen and Sigmund (2018) to allow for singularities with in$\operatorname{dex} 1 / 4$. As an alternative, it might be of interest to extract an explicit quad mesh from the parametrizations produced in this paper. Such an explicit structure would allow the use of graph-based algorithms, which in turn enable the investigation of several challenges, like disconnected or badly aligned structural members, but also stretched iso-contours. Finally, we identify the expansion of the presented approach to 3D as another, highly interesting research area. Besides an increase in computational cost, most of the ideas introduced in this paper can be reused, but we envision challenges such as non-uniqueness of 6-direction field when only 1 layer has a finite width and challenges with the smoothness of the layer-fields as has been discussed in Groen et al. (2020).

\section{Replication of results}

Our work relies on several programming languages (Python, Matlab, $\mathrm{C}++$ ) and also several other codes. Specifically, we use the described homogenization-based topology optimization based on the 88 line MATLAB code by Andreassen et al. (2011). The resulting 4direction fields are combed using the described densitybased search method, and in order to compute the final structures, we use the QuadCover implementation in libigl.

Rather than providing a source code package which would only work under very strict platform requirements, we instead opt to aid the reader in reproducing our results by providing three types of materials:

- An example 4-direction field with associated densities of the type produced for example by the code from Groen and Sigmund (2018)

- A Python script that demonstrates the density-based combing.

- A document outlining the necessary changes that have to be made to the libigl library to make QuadCover work on our type of problems. In particular, this sup- plementary document explains the implementation of the constraint in Equation 6 .

\section{Acknowledgements}

The authors would like to thank professor Niels Aage for discussions and valuable inputs to the project. The authors acknowledge the support of the Villum Fonden through the Villum investigator project InnoTop. The authors would also like to thank Krister Svanberg for providing the MATLAB MMA code.

\section{Conflict of interest}

The authors declare that they have no conflict of interest.

\section{References}

Allaire, G., Geoffroy-Donders, P., Pantz, O., 2018. Topology optimization of modulated and oriented periodic microstructures by the homogenization method. Computers \& Mathematics with Applications .

Allaire, G., Kohn, R., 1993. Optimal-design for minimum weight and compliance in plane-stress using extremal microstructures. European Journal of Mechanics A-solids 12, 839-878.

Andreassen, E., Clausen, A., Schevenels, M., Lazarov, B.S., Sigmund, O., 2011. Efficient topology optimization in matlab using 88 lines of code. Struct. Multidiscip. Optim. 43, 1-16. URL: http: //dx.doi.org/10.1007/s00158-010-0594-7, doi:doi:10.1007/s00158-010-0594-7.

Bendsøe, M.P., 1989. Optimal shape design as a material distribution problem. Structural Optimization 1, 193-202. doi:doi:10.1007/bf01650949.

Bendsøe, M.P., Kikuchi, N., 1988. Generating optimal topologies in structural design using a homogenization method. Computer methods in applied mechanics and engineering 71, 197-224.

Bendsøe, M.P., Sigmund, O., 1999. Material interpolation schemes in topology optimization. Archive of Applied Mechanics 69, 635-654. doi:doi:10.1007/s004190050248.

Bommes, D., Zimmer, H., Kobbelt, L., 2009. Mixedinteger quadrangulation. ACM Transactions On Graphics (TOG) 28, 77.

Bourdin, B., 2001. Filters in topology optimization. International Journal for Numerical Methods in Engineering 50, 2143-2158. doi:doi 10.1002/nme.116. 
Bruns, T., Tortorelli, D., 2001. Topology optimization of non-linear elastic structures and compliant mechanisms. Computer Methods in Applied Mechanics and Engineering 190, 3443-3459. doi:doi:10.1016/S00457825(00)00278-4.

Delmarcelle, T., Hesselink, L., 1994. The topology of symmetric, second-order tensor fields, in: Proceedings of the conference on Visualization'94, IEEE Computer Society Press. pp. 140-147.

Díaz, A., Sigmund, O., 1995. Checkerboard patterns in layout optimization. Structural Optimization 10, 40-45. doi:doi:10.1007/bf01743693.

Donders, P.G., 2018. Homogenization method for topology optmization of struc-tures built with lattice materials. Ph.D. thesis.

Groen, J.P., Sigmund, O., 2018. Homogenization-based topology optimization for high-resolution manufacturable microstructures. International Journal for Numerical Methods in Engineering .

Groen, J.P., Stutz, F.C., Aage, N., Bærentzen, J.A., Sigmund, O., 2020. De-homogenization of optimal multi-scale 3d topologies. Computer Methods in Applied Mechanics and Engineering 364, 112979.

Groen, J.P., Wu, J., Sigmund, O., 2019. Homogenization-based stiffness optimization and projection of $2 \mathrm{~d}$ coated structures with orthotropic infill. Computer Methods in Applied Mechanics and Engineering 349, 722 742. URL: http://www.sciencedirect.com/ science/article/pii/S0045782519301021, doi:doi:10.1016/j.cma.2019.02.031.

Hotz, I., Sreevalsan-Nair, J., Hagen, H., Hamann, B., 2010. Tensor Field Reconstruction Based on Eigenvector and Eigenvalue Interpolation, in: Hagen, H. (Ed.), Scientific Visualization: Advanced Concepts. Schloss Dagstuhl-Leibniz-Zentrum fuer Informatik, Dagstuhl, Germany. volume 1 of Dagstuhl Follow-Ups, pp. 110-123. URL: http:// drops.dagstuhl.de/opus/volltexte/2010/2700, doi:doi: $10.4230 /$ DFU.SciViz.2010.110.

Kälberer, F., Nieser, M., Polthier, K., 2007. Quadcoversurface parameterization using branched coverings, in: Computer graphics forum, Wiley Online Library. pp. $375-384$.

Norris, A.N., 2005. Optimal orientation of anisotropic solids. The Quarterly Journal of Mechanics and Applied Mathematics 59, 29-53.

Pantz, O., Trabelsi, K., 2008. A post-treatment of the homogenization method for shape optimization. SIAM Journal on Control and Optimization 47, 1380-1398.

Pantz, O., Trabelsi, K., 2010. Construction of minimization sequences for shape optimization, in: 2010 15th International Conference on Methods and Models in Automation and Robotics, IEEE. pp. 278-283.

Pedersen, P., 1989. On optimal orientation of orthotropic materials. Structural optimization 1, 101106.

Skiena, S.S., 2008. Sorting and Searching. Springer London, London. pp. 103-144. URL: https: //doi.org/10.1007/978-1-84800-070-4_4, doi:doi:10.1007/978-1-84800-070-4_4.

Svanberg, K., 1987. The method of moving asymptotesa new method for structural optimization. International Journal for Numerical Methods in Engineering 24, 359-373. doi:doi:10.1002/nme.1620240207.

Träff, E., Sigmund, O., Groen, J., 2019. Simple singlescale microstructures based on optimal rank-3 laminates. Structural and Multidisciplinary Optimization 59, 1021-1031.

Vaxman, A., Campen, M., Diamanti, O., Panozzo, D., Bommes, D., Hildebrandt, K., Ben-Chen, M., 2016. Directional field synthesis, design, and processing, in: Computer Graphics Forum, Wiley Online Library. pp. $545-572$. 